\title{
BMJ Open Randomised, prospective, medico- economic nationwide French study of islet transplantation in patients with severely unstable type 1 diabetes: the STABILOT study protocol
}

Sandrine Lablanche, ${ }^{1,2}$ Sandra David-Tchouda, ${ }^{3}$ Jennifer Margier, ${ }^{3}$ Edith Schir, ${ }^{4}$ Anne Wojtusciszyn, ${ }^{5}$ Sophie Borot, ${ }^{6}$ Laurence Kessler, ${ }^{7}$ Emmanuel Morelon, ${ }^{8}$ Charles Thivolet, ${ }^{9}$ François Pattou, ${ }^{10,11,12}$ Marie Christine Vantyghem, ${ }^{12,13}$ Thierry Berney, ${ }^{14}$ Pierre-Yves Benhamou ${ }^{1,2}$

To cite: Lablanche S, DavidTchouda S, Margier J, et al. Randomised, prospective, medico-economic nationwide French study of islet transplantation in patients with severely unstable type 1 diabetes: the STABILOT study protocol. BMJ Open 2017;7: e013434. doi:10.1136/ bmjopen-2016-013434

- Prepublication history for this paper is available online. To view these files please visit the journal online (http://dx.doi.org/10.1136/ bmjopen-2016-013434).

Principal Investigator. $\mathrm{Pr}$ Pierre-Yves Benhamou, Department of Endocrinology, Grenoble University Hospital, CS10217, 38043 Grenoble, France.

Received 13 July 2016 Revised 8 November 2016 Accepted 5 January 2017 CrossMark

For numbered affiliations see end of article.

Correspondence to Dr Sandrine Lablanche; slablanche@chu-grenoble.fr

\section{ABSTRACT}

Introduction: Islet transplantation may be an appropriate treatment option for patients with severely unstable type 1 diabetes experiencing major glucose variability with severe hypoglycaemia despite intensive insulin therapy. Few data are available on the costs associated with islet transplantation in relation to its benefits. The STABILOT study proposes to assess the economic impact of islet transplantation in comparison with the current best medical treatment defined as sensor-augmented pump (SAP) therapy.

Methods: The trial will adopt an open-label, randomised, multicentred design. The study will include 30 patients with severely unstable type 1 diabetes. Eligible participants will be $18-65$ years old, with type 1 diabetes duration $>5$ years, a negative basal or stimulated C-peptide, and severe instability defined by persistent, recurrent and disabling severe hypoglycaemia, despite optimised medical treatment. Participants will be randomised into two groups: one group with immediate registration for islet transplantation, and one group with delayed registration for 1 year while patients receive SAP therapy. The primary endpoint will be the incremental cost-utility ratio at 1 year between islet transplantation and SAP therapy. Perspectives of both the French Health Insurance System and the hospitals will be retained.

Ethics and dissemination: Ethical approval has been obtained at all sites. The trial has been approved by ClinicalTrials.gov (Trial registration ID NCT02854696). All participants will sign a free and informed consent form before randomisation. Results of the study will be communicated during national and international meetings in the field of diabetes and transplantation. A publication will be sought in journals usually read by physicians involved in diabetes care, transplantation and internal medicine.

Trial registration number: NCT02854696;

Pre-results.

\section{Strengths and limitations of this study}

It is the first randomised health economic study performed in islet transplantation.

- It is the first trial to compare islet transplantation with sensor-augmented pump therapy.

- Although the sample size analysed in the trial is low, it is permitted by the expected strong efficacy of islet transplantation.

\section{INTRODUCTION}

Type 1 diabetes mellitus is a chronic disease characterised by the autoimmune destruction of $\beta$ cells resulting, in the absence of treatment, in hyperglycaemia, ketoacidosis and death. Type 1 diabetes mellitus treatment is currently based on multi-daily subcutaneous insulin injections. Some patients with type 1 diabetes develop a particular form of diabetes mellitus with severe instability, previously called brittle diabetes, which is characterised by glucose variability, lack of predictability, unawareness of hypoglycaemic episodes and occurrence of severe hypoglycaemia. Severe hypoglycaemia is associated with alteration in the quality of life, ${ }^{1}$ a 3.2 increased risk of death ${ }^{2} 3$ and an increase in healthcare costs; ${ }^{4}$ glucose variability is associated with a higher risk of microangiopathy progression. ${ }^{5}$ Intensive insulin therapy and use of innovative technologies such as insulin pump therapy and real-time continuous glucose monitoring (RT-CGM) have enabled some patients to reduce their glucose variability and prevent the occurrence of severe hypoglycaemia ${ }^{6}$ other patients, however, fail 
to restore their glucose stability and have persistent severe hypoglycaemia. For such patients, islet transplantation may be a suitable treatment option. Islet transplantation can improve glucose variability, prevent the occurrence of severe hypoglycaemia, enhance glycaemic control $^{78}$ and have a positive impact on quality of life ${ }^{1}$ and on the progression of microangiopathy. ${ }^{9} 10$

Islet transplantation is costly and the question of its cost in relation to its benefits needs to be addressed. Few data are available on islet transplantation costs in relation to the benefits derived. For islet transplantation performed in France and Switzerland, the cost of islet transplantation (including the initial cost and the 1-year follow-up) is estimated at $€ 78000$ and is slightly higher than the cost of whole-pancreas organ transplantation. ${ }^{11}$ Beckwith et $a l^{12}$ performed a health economic evaluation of islet transplantation and showed that transplantation is cost-effective in the short term and cost-saving in the long term when compared with standard insulin therapy: for standard insulin therapy, cumulative cost per patient during a 20-year follow-up was US\$663000 with a cumulative effectiveness of 9.3 QALY (quality-adjusted life years) and an average costeffectiveness ratio of $\$ 71000$ per QALY. For islet transplantation, the cumulative cost was $\$ 519000$ with a cumulative effectiveness of 10.9 QALY and an average cost-effectiveness ratio of $\$ 47800$ per QALY. Nevertheless, the evaluation performed by Beckwith et al was based on estimations and extrapolations from clinical data because actual trial data were lacking. Moreover, current best medical treatment for patients with severely unstable type 1 diabetes is nowadays suggested to be sensor-augmented pump (SAP) therapy comprising continuous subcutaneous insulin infusion integrated with RT-CGM. To the best of our knowledge, no health economic evaluation of islet transplantation has been performed in comparison with SAP therapy. The primary objective of the STABILOT study is to perform a prospective cost-effectiveness analysis to compare islet transplantation versus SAP therapy in patients with severely unstable type 1 diabetes. The main secondary objectives are to assess the clinical and economic benefits of islet transplantation in patients with severe diabetes including short or long-term analysis.

\section{POPULATION AND METHODS}

\section{Study design}

The STABILOT trial is an open-label, prospective, randomised, multicentred trial involving 10 clinical centres in France (Grenoble, Besançon, Clermont-Ferrand, Lille, Lyon, Nantes, Nancy, Montpellier, Paris, and Strasbourg).

\section{Main inclusion criteria}

Patients aged between 18 and 65 years with a duration of type 1 diabetes $>5$ years, with a glycated haemoglobin (HbAlc) $<12 \% \quad$ (HbAlc $<108 \mathrm{mmol} / \mathrm{mol}$ ), insulin requirement $<0.85 \mathrm{UI} / \mathrm{kg} /$ day, negative basal or stimulated C-peptide, and severely unstable type 1 diabetes despite optimised insulin treatment and educational training, will be included. Optimised insulin treatment is defined as pump therapy (or multi-dose insulin injection (MDI) for patients refusing or failing to manage pump therapy). Pump therapy has to be supervised by a clinician who is expert in diabetes management warranting optimal insulin therapy adjustment. Patients have to be educated through their participation in structured psycho-educational programmes, delivered in individual or group settings.

A patient will be considered as experiencing a severely unstable type 1 diabetes if at least two of the following criteria are present: persistence of severe hypoglycaemia defined as the occurrence of at least one episode of severe hypoglycaemia over the previous year; occurrence of ketoacidosis events without obvious aetiology; diagnosis of unaware hypoglycaemic episodes $<3 \mathrm{mmol} / \mathrm{L}$ based on CGM or self-monitoring blood glucose data; a mean blood glucose SD $>50 \%$ or $>40 \mathrm{mg} / \mathrm{dL}$ (2.22 mmol/L) on CGM data; MAGE (mean amplitude of glucose excursions) index $>60 \mathrm{mg} / \mathrm{dL}(3.33 \mathrm{mmol} /$ L); low blood glucose index $>5$; Clarke score $\geq 4$; or HYPOSCORE $>800 .{ }^{13}$

\section{Main exclusion criteria}

- Exclusion criteria related to islet infusion: haemostatic disorders, pre-existing liver disease (plasma ammonia level (PAL), gamma-glutamyl transferase (GGT), aspartate amino transferase/alanine amino transferase $($ ASAT-ALAT) $>2 \mathrm{~N}$ ) or gallbladder lithiasis

- Exclusion criteria related to diabetic complications: evolutive proliferative retinopathy, evolutive nephropathy (glomerular filtration rate $<30 \mathrm{~mL} / \mathrm{min} / 1.73 \mathrm{~m}^{2}$ and/or proteinuria $>0.5 \mathrm{~g}$ /day), evolutive cardiopathy or obliterative arteriopathy with trophic cutaneous lesions

- Exclusion criteria related to immunosuppressant use: haemoglobin $<110 \mathrm{mg} / \mathrm{dL}$ in women and $<120 \mathrm{mg} / \mathrm{dL}$ in men, leuconeutropenia, thrombopenia, systemic infection including chronic hepatitis B, $\mathrm{C}$ and $\mathrm{VIH}$, neoplastic disease and hypertension $>160 / 100 \mathrm{~mm} \mathrm{Hg}$

- Corticoid treatment (except for patients who have benefited from a kidney graft with maintenance steroid therapy)

- Presence of anti-human leucocyte antibodies (anti-HLA) antibody directed against the donor

- Positive B or T cells crossmatch

- Pregnant women, women intending to conceive or breastfeeding woman

\section{Trial intervention and visit schedule}

\section{Pre-inclusion visit}

Participants meeting the inclusion criteria will be invited to give their informed consent. The pre-inclusion visit allows each putative inclusion to be validated via selection and validation procedures by the respective 
committees (ie, paragraphs section committee and validation committee). Once approved by the selection and validation procedures, patients will undergo the inclusion visit.

\section{Inclusion visit}

During the inclusion visit, patients eligible for islet transplantation will be randomised into two parallel groups: the immediate islet transplantation group (IIT group) $(n=15)$ or the delayed islet transplantation group (DIT group) $(\mathrm{n}=15)$. The randomisation will be performed through a web-based central randomisation system and by minimisation. Minimisation aims to ensure that treatment arms are balanced with respect to major confusion factors in the case of low sample size. ${ }^{13}$ However, patients describing life-threatening unstable type 1 diabetes will be directly allocated to immediate islet transplantation without randomisation.

\section{Intervention}

In the IIT group, patients will be immediately registered on the islet transplantation waiting list. When an islet graft becomes available, participants will undergo transplantation. The islet isolation and transplantation procedure as well as the immunosuppressive therapy used in our consortium have been previously described in the GRAGIL Network. ${ }^{7}$ Briefly, pancreases will be obtained from brain-dead multi-organ donors through the Swiss transplant and the French Biomedicine Agency (Agence de la Biomédecine). Islets will be isolated using the Ricordi automated method with local modifications. Islet preparations will be conditioned in gas-permeable transfer bags (Biorep, Miami, Florida, USA) in CMRL 1066 medium supplemented with human albumin (4\%) and heparin ( $35 \mathrm{U} / \mathrm{kg}$ recipient body weight) and transported by ambulance to the transplant centres. Transit times will never exceed 4 hours. The islets will be transplanted intraportally. Patients are scheduled to receive up to a target islet mass of $11000 \mathrm{IEQ} / \mathrm{kg}$ body weight. Consequently, if the first islet infusion does not achieve the $11000 \mathrm{IEQ} / \mathrm{kg}$ body weight threshold, a second and third infusion may be performed, ideally with a time frame of 3 months to achieve the total islet mass. In the IIT group, the reference date for the beginning of the follow-up will be the date of the first islet infusion.

In the DIT group, patients will be registered on the islet transplantation waiting list 1 year after the randomisation. During the delayed period, SAP therapy with predictive low-glucose suspend (threshold $60 \mathrm{mg} / \mathrm{dL}$ ) will be proposed. For patients refusing SAP therapy, a multi-daily injection regimen will be adopted in association with RT-CGM. In the DIT group, the reference date for the beginning of the follow-up will be the date of the inclusion visit.

\section{Follow-up}

In the IIT group, during the waiting period, patients will attend a study visit every 3 months until the islet transplantation procedure is performed. After islet transplantation, protocol requires monthly supervision of the patients by the diabetologist investigator during the first year following first infusion. After year 1, patients will be required to see the diabetologist investigator every 6 months. In the DIT group, patients will be required to see the diabetologist investigator every 3 months during the first year. Complementing the quarterly visits, patients will download pump and CGM data to the clinician on a monthly basis. Based on these data, the clinician can order insulin therapy adjustment through a phone call. At 12 months, the DIT participants group will be registered on the waiting list and will attend for a study visit each 3 months until the islet transplantation procedure. After islet transplantation, the protocol will follow the same pattern as for the IIT group.

In each group and for each visit, a clinical and biological evaluation will be performed as shown in table 1 . Serious adverse events, in particular acute metabolic events (severe hypoglycaemia and ketoacidosis), will be reported prospectively. At 6 and 12 months, a 1-month CGM recording will be performed for each participant. The EuroQol 5 Dimensions (EQ-5D) and the Diabetes Quality of Life (DQoL) questionnaires will be completed as described in table 1 .

\section{Endpoints}

\section{Primary endpoint}

The primary endpoint will be the incremental costeffectiveness ratio at 1 year for islet transplantation versus SAP therapy. Costs will be valued from the perspective of the French healthcare system and hospitals. The effectiveness will be expressed as QALY in a cost-utility analysis. QALY are a composite measure of outcomes where utilities for health states (on 0-1 scale, where 0 corresponds to death and 1 to full health) act as qualitative weights to combine quantity and quality of life. The number of QALY in each group will be assessed with the EQ-5D questionnaire. The EQ-5D measures health status in terms of mobility, self-care, usual activities, pain/discomfort and anxiety/depression.

\section{Secondary endpoints}

The secondary outcomes will allow the investigators to:

1. Assess the cost-effectiveness ratio at 1 year of islet transplantation and SAP therapy for patients with no life-threatening unstable type 1 diabetes. Two criteria of effectiveness will be used: the number of life years gained and the number of severe hypoglycaemia episodes

2. Assess and compare the individual medical benefits in terms of quality of life (DQoL questionnaire), metabolic efficacy, hospitalisations and complications of islet transplantation and SAP therapy at 6 and 12 months

3. Compare the clinical outcomes and costs of patients with life-threatening unstable type 1 diabetes before and after islet cell transplantation 
Table 1 Schedule for visits

\begin{tabular}{|c|c|c|c|c|c|c|c|}
\hline \multirow[b]{2}{*}{ Parameters recorded } & \multicolumn{2}{|l|}{ All } & \multicolumn{2}{|l|}{ IIT group } & \multicolumn{3}{|l|}{ DIT group } \\
\hline & $\begin{array}{l}\text { Pre inclusion } \\
\text { visit }\end{array}$ & $\begin{array}{l}\text { Inclusion } \\
\text { visit }\end{array}$ & $\begin{array}{l}\text { Waiting period } \\
\text { Quarterly visit }\end{array}$ & $\begin{array}{l}\text { Post-transplantation } \\
\text { period } \\
\text { Monthly visit }\end{array}$ & $\begin{array}{l}\text { Delayed period } \\
\text { Quarterly visit }\end{array}$ & $\begin{array}{l}\text { Waiting period } \\
\text { Quarterly visit }\end{array}$ & $\begin{array}{l}\text { Post-transplantation } \\
\text { period } \\
\text { Monthly visit }\end{array}$ \\
\hline \multicolumn{8}{|l|}{ Medical evaluation } \\
\hline Height & $x$ & $x$ & $x$ & $x$ & $x$ & $x$ & $x$ \\
\hline Weight & $x$ & $x$ & $x$ & $x$ & $x$ & $x$ & $x$ \\
\hline Insulin requirement & $x$ & $x$ & $x$ & $x$ & $x$ & $x$ & $x$ \\
\hline Clarke score & $x$ & & & & & & \\
\hline LBGI, MAGE index & $x$ & & & & & & \\
\hline Hyposcore & $x$ & & & & & & \\
\hline Adverse events & & & $x$ & $x$ & $x$ & $x$ & $x$ \\
\hline \multicolumn{8}{|l|}{ Biological evaluation } \\
\hline $\mathrm{HbA1c}$ & $x$ & $x$ & $x$ & $x$ & $\mathrm{x}$ & $x$ & $x$ \\
\hline C-peptide & $x$ & $x$ & & $x$ & & & $x$ \\
\hline Creatinine & $x$ & $x$ & $x$ & $x$ & $x$ & $x$ & $x$ \\
\hline Proteinuria & $x$ & $x$ & $x$ & $x$ & $x$ & $x$ & $x$ \\
\hline Anti-HLA Ab & $x$ & $x$ & $x$ & $x$ & $x$ & $x$ & $x$ \\
\hline Anti-GAD, anti-IA2 Ab & $x$ & $x$ & $x$ & $x$ & $x$ & $x$ & $x$ \\
\hline Complete blood count & $x$ & $x$ & $x$ & $x$ & $\mathrm{x}$ & $x$ & $x$ \\
\hline ASAT/ALAT & $x$ & $x$ & $x$ & $x$ & $x$ & $x$ & $x$ \\
\hline \multicolumn{8}{|l|}{ CGM recording } \\
\hline \multirow[t]{3}{*}{ One-month CGM recording } & & $x$ & & $\mathrm{x}$ & $\mathrm{x}$ & & $\mathrm{x}$ \\
\hline & & & & M6-M12 & M6-M12 & & M6-M12 \\
\hline & & & & post-transplantation & post-inclusion & & post-transplantation \\
\hline \multicolumn{8}{|l|}{ Questionnaire } \\
\hline \multirow[t]{2}{*}{ DQoL } & & $x$ & & $\mathrm{x}$ & $\mathrm{x}$ & & \\
\hline & & & & $\begin{array}{l}\text { M12 } \\
\text { post-transplantation }\end{array}$ & M12 post-inclusion & & \\
\hline EQ-5D & & $x$ & $x$ & $\mathrm{x}$ & $x$ & $x$ & $\mathrm{X}$ \\
\hline & & & & M6-M12 & & & M6-M12 \\
\hline & & & & post-transplantation & & & post-transplantation \\
\hline
\end{tabular}
EQ-5D, EuroQol 5 Dimensions; GAD, glutamic acid decarboxylase; HbA1c, glycated haemoglobin; HLA, human leucocyte antigen; LBGI, low blood glucose index; MAGE, mean amplitude of glucose excursion. 
4. Implement a budget impact analysis

5. Perform a long-term evaluation of the clinical and economic impact of islet transplantation through modelling.

\section{Economic evaluation}

\section{Cost measurement}

To assess the total cost of each group, the number of resources consumed will be prospectively collected for each patient (drugs, medical devices, consultations, transportations, hospitalisation, etc). The French healthcare prices will be used to cost out resources consumed during the follow-up period.

For the procedure costs for islets infusion, the microcosting approach will be used. This approach consists of measuring, by direct observation, all the relevant cost components of the procedure (duration of the procedure, composition of the staff, drugs and medical devices used, type of operating room and the duration of the hospital stays) as variables and costing out each component with the unit production cost or purchasing prices for drugs and medical devices.

\section{QALY estimation}

The EQ-5D will be self-administered at baseline and every 3 months. The utility values are based on the French utility function. ${ }^{14}{ }^{15}$ Utility curves were obtained for each group by plotting average utility values at baseline and every 3 months. The difference in QALY was estimated as the difference in the area between the utility curves for the two groups.

\section{Statistical analysis}

Sample size

The sample size was estimated based upon the primary economic criterion and the secondary clinical criteria based on Glick's ${ }^{15}$ works. Regarding cost-utility analysis, we considered the less favourable following assumptions: difference in costs of $€ 69000 \pm € 50000$ (SD). The average cost for patients with unstable diabetes with DIT was assumed to rise from $€ 6700$ to $€ 25000$ at 12 months (InVS report and data from Beckwith et $a l^{12}$ ). The average cost for patients with unstable diabetes 12 months after islet transplantation was evaluated at between $€ 75000$ (preliminary results from the TRIMECO study) and $€ 95000 .{ }^{12}$ A difference in effects of $0.06 \mathrm{QALY} \pm 0.03,{ }^{12}$ a correlation between difference in costs and effects from -1 to 1 , and a maximum willingness to pay $€ 20000$ per QALY were used. Based on these data, 9-12 patients per group have to be included (calculated using Stata V.11SE). Nevertheless, results on the medico-economic criteria have to be interpreted with caution because of the many assumptions and because of the high instability of the mathematical formula used. Consequently, we also took into account, in the sample size calculation, the clinical hypothesis requiring most of the subjects. We considered a two-tailed $\alpha$ of $5 \%$ and a study power of $90 \%$. Considering a monthly mean (SD) of $25 \pm 20$ hypoglycaemias in the DIT group and $5 \pm 10$ in the IIT group (TRIMECO study preliminary results), it was necessary to include 15 patients per group (calculated using Nquery 6.02 on 31 July 2014).

\section{Analysis}

In this randomised controlled trial, an intention to treat analysis will be performed in line with arguments in the CONSORT statement (http://www.consort-statement. org/). Sociodemographic, clinical and economic data will be analysed per group.

\section{Primary outcome}

The costs and utilities will be estimated for a 1-year horizon. QALY and costs will be described using means (with SDs or $95 \%$ CIs) or medians (with IQRs). Differences in costs and QALY will be described as means (with 95\% CIs) and tested using standard parametric or non-parametric tests (t-test or Mann-Whitney test) as appropriate. The incremental cost-effectiveness ratio will be calculated. To address uncertainty in cost and outcomes across both arms, a sensitivity analysis will be performed.

Missing data will be considered using multiple imputation regression methods.

\section{Secondary outcomes}

1. The incremental cost-effectiveness ratios will be calculated and expressed as incremental cost per life years gained and the number of hypoglycaemia episodes avoided.

2. Comparison of clinical and biological data will be performed, in particular on metabolic events, insulin requirement, hospitalisation or occurrence of complications. Continuous data will be compared using a $\mathrm{t}$-test if the variable was normally distributed or Mann-Whitney test for non-parametric variables. The $\chi^{2}$ test will be used for categorical variables (Fisher's exact test if necessary).

3. Description and comparison of the studied population with life-threatening unstable diabetes based on a data-paired analysis (before-after study) will be performed. Continuous data will be compared using a paired t-test if the variable was normally distributed or Wilcoxon test for non-parametric variables. The MacNemar test will be used for categorical variables (Fleiss test if necessary).

4. The economic burden at 1 and 5 years after islet transplantation in the management of severe forms of type 1 diabetes will be measured. The model will take into account especially the target population, the SAP therapy management cost versus islet transplantation cost, the assumptions about the maintenance or not of insulin-independence over time, and also assumptions about changes in unit costs

5. To simulate the long-term cost, effectiveness and costeffectiveness a Markov model will be used, and we 
will use data from the Stabilot study, from our

TRIMECO cohort and from the literature.

Statistical significance will be considered at a value of $\mathrm{p} \leq 0.05$. All statistical analyses were performed using Stata SE V.12.0 software (StataCorp LP, 4905 Lakeway Drive, College Station, Texas 77845-4512, USA).

\section{STUDY MANAGEMENT}

\section{Selection committee}

A selection committee composed of investigators from each centre will review the medical history and the indication for islet transplantation for each participant being considered for inclusion in the STABILOT protocol. Half of the centres have to be represented in order to authorise the selection procedure. At the end of the selection procedure, the pre-inclusion of the participants is either validated or not.

\section{Validation committee}

The validation committee is an independent committee composed of two members (Professor Penfornis, Diabetologist, Corbeil-Essonnes Hospital, and Dr Schaepelynck-Belicar, Marseille Hospital) in charge to validate the islet transplantation indication and the inclusion in the STABILOT trial for pre-included participants.

\section{Safety}

According to Directive 2001/20/EC, all adverse events will be recorded and reported with the help of the "Terminology Criteria for adverse Events in Trials of adult pancreatic islet transplantation" ${ }^{16}$ All serious adverse events will be reported prospectively to the Sponsor and to the competent authority (ANSM: Agence Nationale de Sécurité du Médicament et des Produits de Santé) and the ethics committee in cases of suspected unexpected serious adverse reactions (SUSAR). Complications related to the islet infusion will be closely monitored as well as adverse events related to the immunosuppressive drugs or concomitant therapy. An independent Data Safety Monitoring Board (DSMB) composed of four experts will be informed of all SUSAR and any safety signals, and will be in receipt of all annual safety reports. The DSMB will report to the Study Management Committee any safety concerns and recommendations for suspension or early termination of the investigation.

\section{Study management and monitoring}

The study coordinator will ensure that the study is conducted in accordance with the International Council for Harmonisation of Technical Requirements for Pharmaceuticals for Human Use (ICH) good clinical practice (GCP) standards through site monitoring visits. A monitoring plan will be written and agreed before first randomisation. An independent data-monitoring committee will monitor $100 \%$ of the data. A datamonitoring report will be edited.

\section{Data management}

Confidentiality of participant data will be observed at all times during the study. Personal details for each participant taking part in the research study and linking them to a unique identification number will be held locally on a study-screening $\log$ in the Trial Master File at each of the investigation centres. All results will remain anonymous. The study identification number will be used on the case report form. Direct access to the source data will be provided for monitoring, audits, ethical committee review and regulatory authority inspections during and after the study as previously described by Leelarathna et al. ${ }^{17}$ Paper copies of the data will be stored for 30 years in line with Public Health Code R. 1123-61.

\section{ETHICAL AND GOVERNANCE APPROVAL}

Ethical approval for this study has been granted by the institutional review board (Person Protection Committee of Grenoble University Hospital ( $\mathrm{n}^{\circ}$ 15-CHUG-14) and Clinical Trial Authorisation has been given by the French National Competent Authority (ANSM): $\mathrm{n}^{\circ}$ idRCB 2015-00350-49. The trial has been approved by ClinicalTrials.gov (Trial registration ID NCT02854696). Each important protocol modification will be communicated to the Person Protection Committee, to ANSM, to ClinicalTrials.gov and to each study centre.

\section{AGENDA}

Screening and recruitment began in June 2016 and the study will be completed by winter 2020 .

\section{Author affiliations}

${ }^{1}$ Department of Diabetology, Pôle DigiDune, Grenoble University Hospital, Grenoble Alpes University, Grenoble, France

${ }^{2}$ Grenoble Alpes University, INSERM, U1055Laboratory of Fundamental and Applied Bioenergetics, Grenoble, France

${ }^{3}$ Cellule d'évaluation médico-économique des innovations, $\mathrm{CHU}$ Grenoble Alpes, CIC 1406 Grenoble, TIMC-Imag UMR 5525 Univ. Grenoble Alpes, Grenoble, France

${ }^{4}$ Centre régional de Pharmacovigilance, Grenoble, France

${ }^{5}$ Centre Hospitalier de Montpellier, Pôle Rein Hypertension Métabolisme, Service d'Endocrinologie, Montpellier, France

${ }^{6}$ Centre Hospitalier Universitaire Jean Minjoz, Service d'EndocrinologieMétabolisme et Diabétologie-Nutrition, Besançon, France

${ }^{7}$ Hôpitaux Universitaires de Strasbourg, Service d'Endocrinologie Diabète et Maladies Métaboliques, Pôle NUDE, Strasbourg, France

${ }^{8}$ Hospices Civils de Lyon, Service d'Urologie et de Chirurgie de la Transplantation, Pôle Chirurgie, Lyon, France

${ }^{9}$ Hospices Civils de Lyon, Service de Diabétologie-Endocrinologie-Maladies Métaboliques et de Chirurgie de la Transplantation, Pôle Chirurgie, Lyon, France

${ }^{10}$ University Lille, CHRU Lille, Inserm U1190 Translational research for diabetes, Lille, France

${ }^{11}$ European Genomic Institute for Diabetes, EGID, Lille, France

${ }^{12}$ Department of Endocrine Surgery, Hôpital Huriez Lille University Hospital, Lille Cedex, France

${ }^{13}$ Department of Endocrinology and Metabolism, Hôpital Huriez, Lille University Hospital, Lille Cedex, France

${ }^{14}$ Departement of Surgery, Islet Isolation, and Transplantation Center, Geneva University Hospitals, Geneva, Switzerland 
Acknowledgements The abstract has been published as a poster for the 17th Congress of the European Society for Organ Transplantation 13-16 September 2015, Brussels, Belgium.

Collaborators The STABILOT Trial Investigators-Main centres: Grenoble University Hospital: Pierre Yves Benhamou, Sandrine Lablanche, Rachel Tetaz; Besançon University Hospital: Sophie Borot; Clermont-Ferrand University Hospital: Igor Tauveron, Béatrice Roche; Geneva University Hospital: Thierry Berney; Lille University Hospital: François Pattou, Marie-Christine Vantyghem, Kanza Benomar, Christian Noel; Lyon University Hospital: Charles Thivolet, Emmanuel Morelon, Lionel Badet, Fanny Buron; Montpellier University Hospital: Anne Wojtusciszyn, Eric Renard; Nancy University Hospital: Luc Frimat, Sophie Girerd; Nantes University Hospital: Diego Cantarovich, Lucy Chaillous; Paris University Hospital: Pierre Cattan, Jean-Pierre Riveline, Marie-Noelle Peraldi, Olivier Bourron; Strasbourg University Hospital: Laurence Kessler, François Moreau, Philippe Baltzinger et Thibault Bahougne. Islet Production Centres: Geneva: Domenico Bosco, Nadine Pernin; Grenoble: Harald Egelhofer, Anaick Moisan, Virginie Persoons; Lille: Julie Kerr-Conte, Valery Gmyr, Rimed Ezzouaoui; Paris: Mathieu Armanet.

Contributors All the authors participated in the research design. SL, SD-T, JM, ES, LK, MCV, FP, P-YB participated in the writing paper.

Funding The STABILOT protocol (V.2.3, 14/12/2015) was supported by grants from Projet de Recherche Medico Economique National 2014, DGOS (Grant number 14-0225) and by grant no. 3200B0-102134 from the Swiss National Foundation for Scientific Research.

Competing interests None declared.

Patient consent Obtained.

Provenance and peer review Not commissioned; externally peer reviewed.

Open Access This is an Open Access article distributed in accordance with the Creative Commons Attribution Non Commercial (CC BY-NC 4.0) license, which permits others to distribute, remix, adapt, build upon this work noncommercially, and license their derivative works on different terms, provided the original work is properly cited and the use is non-commercial. See: http:// creativecommons.org/licenses/by-nc/4.0/

\section{REFERENCES}

1. Benhamou PY, Milliat-Guittard L, Wojtusciszyn A, et al., GRAGIL group. Quality of life after islet transplantation: data from the GRAGIL 1 and 2 trials. Diabet Med 2009;26:617-21.
2. Tanenberg RJ, Newton CA, Drake AJ. Confirmation of hypoglycemia in the "dead-in-bed" syndrome, as captured by a retrospective continuous glucose monitoring system. Endocr Pract 2010;16:244-8.

3. McCoy RG, Van Houten HK, Ziegenfuss JY, et al. Increased mortality of patients with diabetes reporting severe hypoglycemia. Diabetes Care 2012;35:1897-901.

4. Hammer M, Lammert M, Mejias SM, et al. Costs of managing severe hypoglycaemia in three European countries. J Med Econ 2009;12:281-90.

5. Retinopathy and nephropathy in patients with type 1 diabetes four years after a trial of intensive therapy. Am J Ophthalmol 2000;129:704-5.

6. Bergenstal RM, Klonoff DC, Garg SK, et al., ASPIRE In-Home Study Group. Threshold-based insulin-pump interruption for reduction of hypoglycemia. N Engl J Med 2013;369:224-32.

7. Lablanche S, Borot S, Wojtusciszyn A, et al., GRAGIL Network Five-year metabolic, functional, and safety results of patients with type 1 diabetes transplanted with allogenic islets within the Swiss-French GRAGIL network. Diabetes Care 2015;38:1714-22.

8. Barton FB, Rickels MR, Alejandro R, et al, Improvement in outcomes of clinical islet transplantation: 1999-2010. Diabetes Care 2012;35:1436-45

9. Thompson DM, Meloche M, Ao Z, et al. Reduced progression of diabetic microvascular complications with islet cell transplantation compared with intensive medical therapy. Transplantation 2011;91:373-8.

10. Warnock GL, Thompson DM, Meloche RM, et al. A multi-year analysis of islet transplantation compared with intensive medical therapy on progression of complications in type 1 diabetes. Transplantation 2008;86:1762-6.

11. Guignard AP, Oberholzer J, Benhamou PY, et al., GRAGIL Group Cost analysis of human islet transplantation for the treatment of type 1 diabetes in the Swiss-French Consortium GRAGIL. Diabetes Care 2004:27:895-900.

12. Beckwith J, Nyman JA, Flanagan B, et al. A health economic analysis of clinical islet transplantation. Clin Transplant 2012;26:23-33.

13. Scott NW, McPherson GC, Ramsay CR, et al. The method of minimization for allocation to clinical trials. A review. Control Clin Trials 2002;23:662-74.

14. Chevalier J, de Pouvourville G. Valuing EQ-5D using time trade-off in France. Eur J Health Econ 2013;14:57-66.

15. Glick HA. Sample size and power for cost-effectiveness analysis (part 1). Pharmacoeconomics 2011;29:189-98.

16. Consortium CIT. Terminology criteria for adverse events in trials of adults pancreatic islet transplantation, 2011. http://www.isletstudy. org/CITDocs/CIT-TCAE\%20V5.0_03Aug2011.pdf

17. Leelarathna L, Dellweg S, Mader JK, et al., AP @ home consortium. Assessing the effectiveness of 3 months day and night home closed-loop insulin delivery in adults with suboptimally controlled type 1 diabetes: a randomised crossover study protocol. BMJ Open 2014;4:e006075. 